\title{
Spontaneous Superficial Temporal Artery Pseudoaneurysm in Elderly Women -Three Case Reports-
}

\author{
Kouhei NiI, ${ }^{1}$ Masanori TSUTSUMI, ${ }^{1}$ Hiroshi AIKAWA, ${ }^{1}$ Masanari ONIZUKA, ${ }^{1}$ \\ Kimiya SAKAMOTO, ${ }^{1}$ Ritsuro INOUE, ${ }^{1}$ Hiroya NAKAU,${ }^{1}$ Minoru IKO, ${ }^{1}$ \\ Seiji HARAOKA, ${ }^{2}$ Fumihiko HARA, ${ }^{3}$ and Kiyoshi KAZEKAWA ${ }^{1}$
}

Departments of ${ }^{1}$ Neurosurgery and

${ }^{2}$ Pathology, Fukuoka University Chikushi Hospital, Chikushino, Fukuoka;

${ }^{3}$ Department of Neurology, Hara Hospital, Onojo, Fukuoka

\begin{abstract}
Three women older than 75 years presented with spontaneous superficial temporal artery (STA) pseudoaneurysms manifesting as a pulsatile mass in the preauricular region. None of the patients had a history of trauma. Histological examination of the surgically removed masses identified pseudoaneurysms based on the presence of connective tissue and adventitia. Spontaneous STA pseudoaneurysms are extremely rare. We suggest that all 3 aneurysms were associated with latent dissection and external force exerted by the frames of glasses.
\end{abstract}

Key words: spontaneous pseudoaneurysm, superficial temporal artery, elderly woman, glasses frame

\section{Introduction}

Superficial temporal artery (STA) aneurysms are relatively uncommon, and were first reported by Bartholin in 1740.4) STA aneurysms are commonly associated with trauma, ${ }^{3,12,14)}$ and primarily occur in young males as pseudoaneurysms. ${ }^{7,10,13)}$ STA aneurysms may present as a pulsatile mass, throbbing headache, or vascular murmur. The natural history has not been well established, and as rupture in the course of aneurysm progression is possible, ${ }^{8)}$ radical treatment of these aneurysms has been recommended.9) Spontaneous STA pseudoaneurysms not associated with trauma are extremely rare. ${ }^{3)}$ We treated 3 elderly women with no history of trauma who presented with STA aneurysms identified as pseudoaneurysms.

\section{Case Reports}

Case 1: A 75-year-old woman had noticed a pulsatile mass in the right preauricular region 6 months prior to presenting at our hospital. She had no relevant past history including head injury. She had hypertension and hypercholesterolemia and had worn glasses since childhood for myopia. Physical examination revealed a $15-\mathrm{mm}$ pulsatile mass with bruit in the right preauricular region. The mass was painless and she had no tinnitus. Neurological exami-

Received July 7, 2010; Accepted March 9, 2011

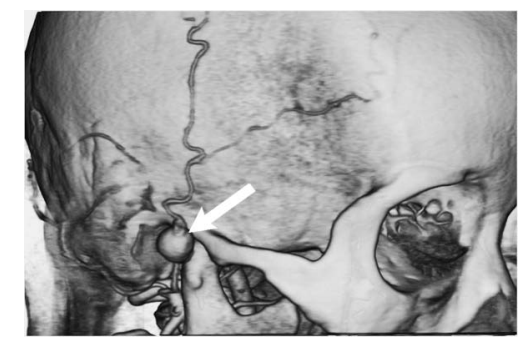

Fig. 1 Case 1. Three-dimensional computed tomography angiogram (volume rendering image) showing an aneurysm at the origin of the superficial temporal artery (arrow).

nation found no abnormalities. Her blood count and routine biochemical and serological studies were within normal limits. Head computed tomography (CT) angiography revealed a fusiform aneurysm arising from the main trunk of the right STA (Fig. 1). The aneurysm was $16 \times 15 \mathrm{~mm}$ in diameter and located lateral to the zygomatic arch. The border between the aneurysm neck and the main trunk of the STA was unclear. The aneurysm was excised at her request. A linear skin incision was performed, and the distal and proximal regions of the aneurysm were ligated for removal (Fig. 2). The postoperative course was uneventful with no recurrence of the aneurysm. The histological diagnosis was pseudoaneurysm based on disruption of the in- 

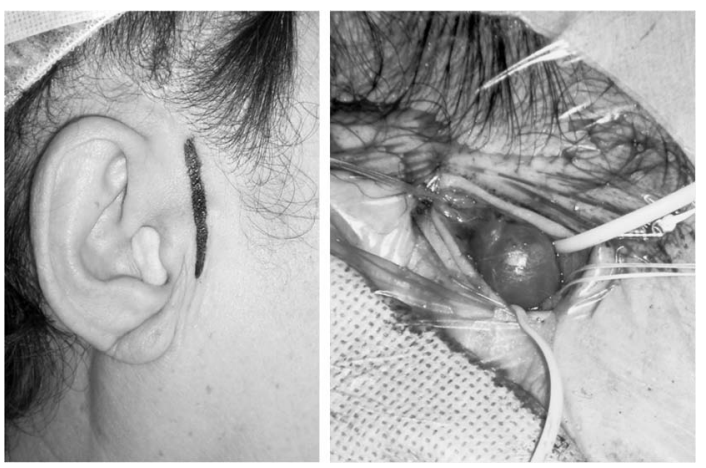

Fig. 2 Case 1. Left: Preoperative photograph showing a mass in the preauricular region. Right: Intraoperative photograph showing the superficial temporal artery aneurysm and the proximal and distal parent artery.
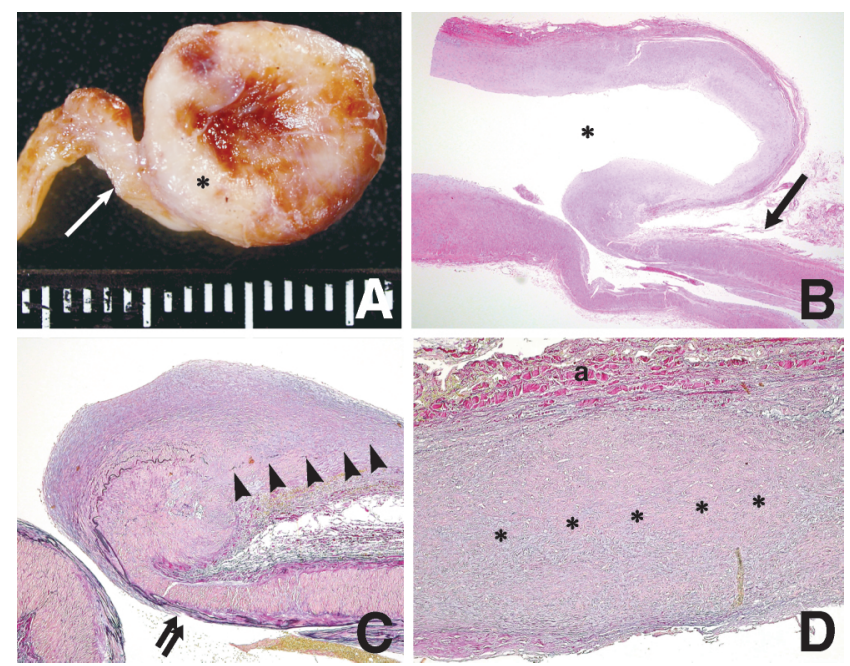

Fig. 3 Case 1. A: Photograph of the surgical specimen showing an aneurysm (asterisk) and the superficial temporal artery (arrow). B: Photomicrograph of the surgical specimen showing an aneurysm (asterisk) and the normal superficial temporal artery (arrow). Hematoxylin and eosin stain, original magnification $\times 2$. C: Photomicrograph showing disruption of the internal elastic lamina (arrowheads). The internal elastic lamina of the superficial temporal artery close to the aneurysm is clearly identified (double arrows). Victoria blue-hematoxylin and eosin stain, original magnification $\times 4$. D: Photomicrograph showing the aneurysm wall consists of connective tissue (asterisks) and adventitia (a). There are no smooth muscle cells or inflammatory cells. Victoria blue-hematoxylin and eosin stain, original magnification $\times 10$.

ternal elastic lamina, and presence of connective tissue and adventitia without intima and media (Fig. 3). There was no evidence of inflammation.

Case 2: A 76-year-old woman had progressive chemosis and conjunctiva. She wore glasses and was hospitalized with a diagnosis of spontaneous dural carotid cavernous fistula (CCF). She had symptoms caused by a CCF and presented with a pulsatile mass in the left preauricular

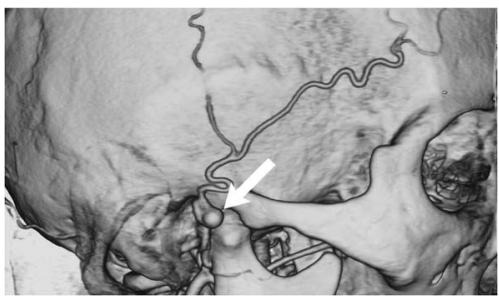

Fig. 4 Case 3. Three-dimensional computed tomography angiogram (volume-rendering image) showing an aneurysm at the origin of the right superficial temporal artery (arrow).

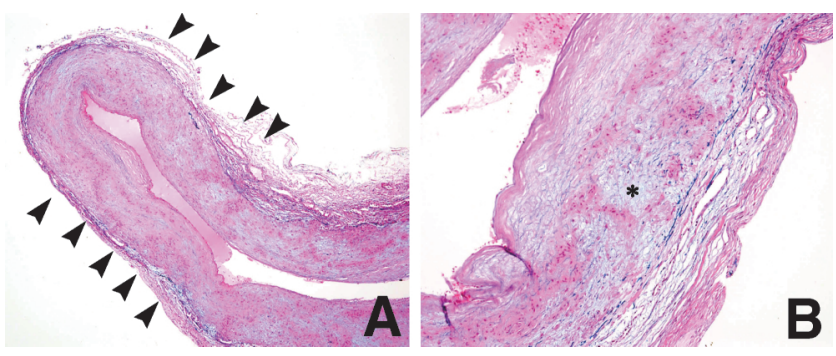

Fig. 5 Case 3. A: Photomicrograph of the surgical specimen showing that the aneurysm consists of connective tissue and adventitia, and the elastic membrane is fragmented (arrowheads). Victoria blue-hematoxylin and eosin stain, original magnification $\times 4$. B: There are a few smooth muscle cells in the aneurysm wall (asterisk). Victoria blue-hematoxylin and eosin stain, original magnification $\times 10$.

region. She had a history of hypertension, diabetes, and hypercholesterolemia, but no history of head injury. Preoperative cerebral angiography showed a STA aneurysm and a CCF involving the internal maxillary and accessory meningeal arteries. Endovascular treatment (transarterial and transvenous embolization) for the CCF resulted in permanent cure. We excised the aneurysm at her request. The histological diagnosis was pseudoaneurysm based on the presence of connective tissue and adventitia without intima, media, and elastic lamina.

Case 3: A 76-year-old woman was hospitalized for the treatment of a right middle cerebral artery aneurysm. She wore glasses and had noticed a pulsatile mass in the right preauricular region. Her past history included hypertension and hypercholesterolemia. Physical examination detected a $10-\mathrm{mm}$ mass in the right preauricular region. The mass was painless and she had no tinnitus. Neurological examination detected no abnormalities. Blood count and biochemical examinations were within normal limits. CT angiography showed a fusiform STA aneurysm (Fig. 4). She underwent excision of the STA and clipping of the cerebral aneurysm in one procedure. The histological diagnosis was pseudoaneurysm (Fig. 5).

\section{Discussion}

The STA has a relatively exposed superficial course under the skin against the hard skull, so STA aneurysms are at- 
tributed primarily to trauma and usually occur in young males as a consequence of head trauma. ${ }^{3,7,10,12,13)}$ Histological examination of most STA aneurysms reveals disruption of the internal elastic lamina and the presence of fibrous connective tissue and hematoma, indicative of pseudoaneurysm. ${ }^{2,5,8,15)}$ Spontaneous STA aneurysms, in contrast, tend to be associated with arteriosclerosis, are characterized by atherosclerotic plaques and calcification, and most are identified as true aneurysms by histological examination. ${ }^{1,6,11,14)}$ Although we first thought that the STA aneurysms in our elderly patients were attributable to arteriosclerosis, we identified them as pseudoaneurysms based on the histological examination of the aneurysm wall revealing disruption of the internal elastic lamina and presence of the connective tissue and adventitia only. ${ }^{16}$ Spontaneous STA pseudoaneurysms in the absence of trauma are extremely rare, with only one previously reported case. ${ }^{3)}$

The pathogenesis of spontaneous STA pseudoaneurysm remains poorly understood. We have identified two possible pathogenetic mechanisms. The first mechanism is spontaneous STA dissection. ${ }^{3}$ However, in our patients, histological examination identified no salient features indicative of dissection, as the prolonged time course from aneurysm formation may result in repair of the dissection plane. The second pathogenetic mechanism involves minor trauma. None of our patients had a history of head trauma, but all wore glasses and the aneurysm developed in an area just below the frame of the glasses in each patient. Therefore, external force causing compression or friction on the STA by the tight frame of the glasses may be associated with hemodynamic stress and critical damage to the vessel wall, and result in the development of STA pseudoaneurysm.

The differential diagnosis may include arteriovenous fistula, neoplasm of the facial nerves and the parotid gland, inflammatory lesions such as temporal arteritis, Marfan's syndrome, and fibromuscular alloplasia. ${ }^{3,12)}$ STA aneurysms can be identified relatively easily with diagnostic imaging techniques such as CT, magnetic resonance imaging, and ultrasonography. In our patients, CT angiography was effective and minimally invasive, and provided important information on the anatomical relationship between the aneurysm and surrounding tissue. In the surgical treatment of aneurysms in the proximal portion of the STA, the relationship between the aneurysm and the zygomatic arch is important and CT angiography can clearly demonstrate the topographic relationship. ${ }^{6)}$

The present three cases of STA pseudoaneurysms in elderly patients were associated with latent dissection and external force exerted by the tight frames of glasses. Surgical resection of the STA aneurysms produced satisfactory outcomes in all 3 patients.

\section{Acknowledgment}

We thank Ms. Ursula Petralia for assistance in preparing this article.

\section{References}

1) Endo T, Mori $K$, Maeda $M$ : Multiple arteriosclerotic fusiform aneurysms of the superficial temporal artery-case report. Neurol Med Chir (Tokyo) 40: 321-323, 2000

2) Fernández-Portales I, Cabezudo JM, Lorenzana L, Gómez L, Porras L, Rodríguez JA: Traumatic aneurysm of the superficial temporal artery as a complication of pin-type head-holder device. Case report. Surg Neurol 52: 400-403, 1999

3) Fujii S, Kajikawa H, Yamamura K, Wada M, Shimamoto F: [Spontaneous dissecting aneurysm of the superficial temporal artery: a case report]. No Shinkei Geka 23: 797-800, 1995 (Japanese)

4) Han K, Borah GL: Pseudoaneurysm of the anterior superficial temporal artery. Ann Plast Surg 27: 650-653, 1996

5) Higashino T, Kawashima M, Mannoji H: Three-dimensional computed tomography angiography for the investigation of superficial temporal artery pseudoaneurysms-two case reports-. Neurol Med Chir (Tokyo) 45: 152-155, 2005

6) Kawabori M, Kuroda S, Nakayama N, Kenmotsu Y, Shimizu $\mathrm{H}$, Tanino M, Iwasaki Y: Spontaneous giant aneurysm of the superficial temporal artery: case report. Neurol Med Chir (Tokyo) 49: 198-201, 2009

7) Levisianos I, Sood V: Traumatic aneurysm (pseudoaneurysm) of the superficial temporal artery. Emerg Med J 25: 239-240, 2008

8) Myer CM III, Donegan JO: Traumatic aneurysm of the proximal superficial temporal artery. Head Neck Surg 5: 181-185, 1982

9) Nishioka T, Kondo A, Aoyama I, Nin K, Shimotake K, Tashiro H, Takahashi J, Kusaka H: [A case of spontaneous superficial temporal artery aneurysm]. No Shinkei Geka 16: 1009-1012, 1988 (Japanese)

10) Peick A, Nichols WK, Curtis JJ, Silver D: Aneurysms and pseudoaneurysms of the superficial temporal artery caused by trauma. J Vasc Surg 8: 606-610, 1988

11) Riaz AA, Ismail M, Sheikh N, Ahmed N, Atkin G, Richman P, Loh A: Spontaneously arising superficial temporal artery aneurysms: a report of two cases and review of the literature. Ann R Coll Surg Engl 86: W38-40, 2004

12) Schechter MM, Gutstein RA: Aneurysms and arteriovenous fistula of the superficial vessels. Radiology 97: 549-557, 1970

13) Sirin G, Bolcal C, Akay T, Iyem H, Tatar H: [Traumatic aneurysm of the superficial temporal artery: a case report]. Ulus Travma Acil Cerrahi Derg 13: 60-62, 2007 (Turkish)

14) Uchida N, Sakuma M: Atherosclerotic superficial temporal artery aneurysm: report of a case. Surg Today 29: 575-578, 1999

15) Walker MT, Liu BP, Salehi SA, Badve S, Batjer HH: Superficial temporal artery pseudoaneurysm: diagnosis and preoperative planning with CT angiography. AJNR Am J Neuroradiol 24: 147-150, 2003

16) Weller CB, Reeder C: Traumatic pseudoaneurysm of the superficial temporal artery: two cases. J Am Osteopath Assoc 101: 284-287, 2001

Address reprint requests to: Kiyoshi Kazekawa, MD, Department of Neurosurgery, Fukuoka University Chikushi Hospital, 1-1-1 Zokumyoin, Chikushino, Fukuoka 818-8502, Japan. e-mail: kazekawa@xb3.so-net.ne.jp 